\title{
Judicialização da saúde nos municípios da região metropolitana de
}

\section{Belém-PA}

Judicialization of health in the municipalities of the metropolitan region of Belém-PA

Judicialización de la salud en los municipios de la región metropolitana de Belém-PA

Taissa Viana Damasceno ${ }^{1}$

Krishina Day Carrilho Bentes Ribeiro²

\begin{abstract}
Resumo
Objetivo: apresentar o panorama municipal das solicitações dos processos judiciais referentes a medicamentos a partir das observações nos municípios da região metropolitana de Belém-PA; verificar quais os argumentos utilizados pelos autores, juízes e réus em observância da compatibilidade dos processos judiciais com a política pública de medicamentos; e classificar os medicamentos solicitados nessas ações. Metodologia: pesquisa realizada a partir do projeto $A$ judicialização da política pública de saúde nos municípios brasileiros, realizada no segundo semestre de 2017, nos municípios de Belém, Marituba e Benevides. Estudo descritivo de abordagem quantitativa com demandas relacionadas à saúde, por meio da análise de 264 processos de ações judiciais, em que os dados encontrados foram organizados em uma planilha feita no software Excel 2010. Resultados: do total de processos analisados, 40 são referentes a medicamentos, totalizando 72 fármacos diferentes, estes foram divididos em presentes nas Relações de medicamentos essenciais (46), ausentes nas listas do SUS (25) e os que não possuem registro para uso (1). O Ministério Público e Defensoria Pública são os atores mais procurados pela sociedade na mediação de conflitos e suas resoluções. Os autores das ações, fazem uso do arcabouço legal relacionado a saúde para justificar seus argumentos. Conclusão: a solicitação de medicamentos não presentes nas listas dos SUS mostra a falta de conhecimento dos profissionais prescritores, visto que, entre os medicamentos prescritos, existe uma opção igual nas relações de medicamentos essenciais e que não causará risco ao paciente.
\end{abstract}

Palavras-chave: Judicialização da saúde. Sistemas de saúde. Terapêutica.

\begin{abstract}
Objective: to present a panorama of the requests for judicial proceedings concerning medicines from the observations in the municipalities of the metropolitan region of Belém-PA; verify the arguments used by the plaintiffs, judges and defendants in observance of the compatibility of court proceedings with public drug policy; and classify the medications requested in these actions. Methodology: research conducted from the project The judicialization of public health policy in Brazilian municipalities, conducted in the second half of 2017, in the municipalities of Belém, Marituba and Benevides. Descriptive study of quantitative approach with health-related demands, through the analysis of 264 lawsuits, in which the data found were organized in a spreadsheet made in Excel 2010 software. Results: 40 out of the total number of cases analyzed refer to totaling 72 different drugs,
\end{abstract}


these were divided into those present in the Essential Drug Relationships (46), absent from the SUS lists (25) and those not registered for use (1). The Public Prosecution Service and the Public Defender's Office are the most sought after actors in conflict mediation and their resolutions. The plaintiffs use the health-related legal framework to justify their arguments. Conclusion: the request for drugs not on the SUS lists shows the lack of knowledge of prescribing professionals, since among the prescribed drugs, there is an equal choice in the relationships of essential drugs and that will not cause risk to the patient.

Keywords: Health's Judicialization. Health Systems. Therapeutics.

\section{Resumen}

Objetivo: presentar el panorama municipal de las solicitudes de procedimientos judiciales sobre medicamentos a partir de las observaciones en los municipios de la región metropolitana de Belém-PA; verificar los argumentos utilizados por los demandantes, los jueces y los acusados en cumplimiento de la compatibilidad de los procedimientos judiciales con la política pública de drogas; y clasificar los medicamentos solicitados en estas acciones. Metodología: investigación realizada desde el proyecto La judicialización de la política de salud pública en los municipios brasileños, realizada en el segundo semestre de 2017, en los municipios de Belém, Marituba y Benevides. Estudio descriptivo de un enfoque cuantitativo con demandas relacionadas con la salud, a través del análisis de 264 juicios, en el que los datos encontrados se organizaron en una hoja de cálculo realizada en el software Excel 2010. Resultados: 40 del total de casos analizados se refieren a totalizando 72 medicamentos diferentes, estos se dividieron en aquellos presentes en las Relaciones de medicamentos esenciales (46), ausentes de las listas de SUS (25) y aquellos que no están registrados para su uso (1). El Servicio de la Fiscalía y la Oficina del Defensor Público son los actores más buscados en la mediación de conflictos y sus resoluciones. Los demandantes utilizan el marco legal relacionado con la salud para justificar sus argumentos. Conclusión: la solicitud de medicamentos que no están en las listas del SUS muestra la falta de conocimiento de los profesionales que los recetan, ya que entre los medicamentos prescritos hay una opción equitativa en las relaciones de los medicamentos esenciales y eso no causará riesgo para el paciente.

Palabras-Ilave: Judicialización de la Salud. Sistemas de Salud. Terapéutica.

\section{Introdução}

O Sistema Único de Saúde (SUS) define a saúde como sendo um direito fundamental do ser humano e de responsabilidade do Estado, e este deve formular e executar políticas econômicas e sociais que visem à redução de riscos de doenças e de outros agravos, além de estabelecer condições que assegurem acesso universal e igualitário às ações e aos serviços para a promoção, proteção e recuperação da saúde (1).

Entre os princípios doutrinários que norteiam o SUS, estão: a universalidade, equidade, integridade, regionalização e hierarquização, resolubilidade e descentralização. Todos têm por objetivo garantir a atenção à saúde a qualquer cidadão, tanto na realização de ações de vigilância epidemiológica, sanitária, controle de vetores e educação, quanto na 
promoção, proteção e recuperação da saúde. As responsabilidades para a aplicação dessas ações e serviços estão divididas entre os vários níveis de governo e seus órgãos: no âmbito da União, pelo Ministério da Saúde; no âmbito dos estados e do Distrito Federal, pela respectiva Secretaria de Saúde ou órgão equivalente; e no âmbito dos municípios, pela respectiva Secretaria de Saúde ou órgão equivalente.

Além da política que norteia o SUS, foram criadas a Política Nacional de Medicamentos (PNM) e a Política Nacional de Assistência Farmacêutica (PNAF) com o intuito de promover a melhoria das condições da assistência à saúde da população. A primeira, criada em 1998 e sendo parte essencial da Política Nacional de Saúde (PNS), foi elaborada para garantir o acesso da população a medicamentos seguros, eficazes e de qualidade, e para promover seu uso racional (2). Inicialmente denominada de Assistência Farmacêutica (AF), a PNAF foi criada a partir da instituição da Central de Medicamentos (CEME), que tinha o papel de fornecer medicamentos à população que não possuía condições financeiras. Sendo essa política determinante para a resolubilidade da atenção e dos serviços em saúde que envolve a alocação de grandes volumes de recursos públicos (3), com a Resolução no 338 do Conselho Nacional de Saúde, de 6 de maio de 2004, a AF passou a ser a Política Nacional de Assistência Farmacêutica, que se caracteriza como:

Conjunto de ações voltadas à promoção, proteção e recuperação da saúde, tanto individual como coletiva, tendo o medicamento como insumo essencial e visando ao acesso e ao seu uso racional. Este conjunto envolve a pesquisa, o desenvolvimento e a produção de medicamentos e insumos, bem como a sua seleção, programação, aquisição, distribuição, dispensação, garantia da qualidade dos produtos e serviços, acompanhamento e avaliação de sua utilização, na perspectiva da obtenção de resultados concretos e da melhoria da qualidade de vida da população (4).

Com a publicação da Portaria GM o 204/2007, o financiamento da Assistência Farmacêutica está sob responsabilidade dos três gestores do SUS e é dividido em Atenção Básica; Atenção de Média e Alta Complexidade Ambulatorial e Hospitalar; Vigilância em Saúde; Assistência Farmacêutica; Gestão do SUS e Investimentos na Rede de Serviços de Saúde (5). Sobre seu planejamento da AF tem-se o objetivo de se ter uma visão mais ampliada e um melhor conhecimento dos problemas internos e externos presentes, gerenciar objetivos e buscar resultados, proporcionar eficiência, eficácia e efetividade nas ações programadas e possibilitar o controle, o aperfeiçoamento contínuo, a avaliação permanente das ações e resultados alcançados (5). 
Para a garantia de seus direitos, principalmente aqueles voltados à assistência farmacêutica, a população faz uso de recursos denominados de demandas judiciais, que se formam a partir de um processo valido e regular instaurado na Justiça de $1^{\circ} \mathrm{Grau}$, em nível Estadual. O processo é uma manifestação de um direito da pessoa humana (6) e todo pedido jurídico deverá ser examinado pelo juiz de 1ํㅡrau, baseando-se em requisitos processuais, para a sua validação. Levando-se em consideração o princípio do devido processo legal e o princípio do contraditório, primeiro o juiz analisa as partes em conflito, para depois, a partir de decisões diversificadas, ouvir as partes que levam a ele as provas de suas alegações. Cabe ao juiz se aproximar da verdade dos fatos, produzindo provas de ofício. Além disso, ele deve analisar alguns requisitos essenciais do pedido do autor do pedido: se há verossimilhança dos fatos, ou seja, se a prova apresentada possui indícios de ser verdadeira; se há perigo da demora da prestação jurisdicional, ou seja, se o juiz autorizar o fornecimento após a instrução de todo procedimento judicial (na sentença), esta demora pode resultar em violação irreparável do direito do autor; e se o pedido do autor está amparado por lei (7).

As demandas judiciais podem ser agrupadas em duas categorias: as justificadas e as injustificadas. As justificadas caracterizam-se por aquelas solicitações de medicamentos previstos nas listas do SUS - Relação Nacional de Medicamentos Essenciais (Rename) para o fornecimento público, mas que possuem seu acesso prejudicado por problemas de gestão dos serviços farmacêuticos; protocolos restritivos ou desatualizados; e medicamentos não incorporados às listas, mas com boas evidências de seu benefício e que representam uma nova alternativa quando os medicamentos disponíveis não podem ser empregados.

Já as demandas não justificadas são aquelas em que os medicamentos não possuem o devido registro na Agência Nacional de Vigilância Sanitária (Anvisa); medicamentos não incorporados, mas com alternativa terapêutica disponível no SUS; e medicamentos cuja literatura científica não recomenda a indicação para a qual foram prescritos (8).

Durante a Conferência da Organização Mundial da Saúde de Alma Ata, realizada em 1978, definiu-se saúde como sendo:

O estado de completo bem-estar físico, mental e social, e não simplesmente a ausência de doença ou enfermidade, é um direito fundamental e a consecução do mais alto nível possível de saúde é a mais importante meta social mundial, cuja realização requer a ação de muitos setores sociais e econômicos, além do setor de saúde (9). 
A Constituição Federal de 1988, em seu artigo 196, define saúde como sendo:

direito de todos e dever do Estado, garantido mediante políticas sociais e econômicas que visem à redução do risco de doença e de outros agravos e ao acesso universal e igualitário às ações e serviços para sua promoção, proteção e recuperação.(10).

Para exercer tal direito, o Estado necessita primeiramente de políticas públicas, que está dividida entre os poderes Legislativo, que tem o poder de elaborar as leis; o Executivo, que define as prioridades e escolhe os meios para a execução; e o Judiciário, que fiscaliza a aplicação das leis e garante a proteção aos direitos enumerados na Constituição Federal.

As tarefas sociais têm seus fundamentos nas próprias normas constitucionais sobre direitos sociais; a sua observação pelo Legislativo e Executivo pode e deve ser controlada pelo Judiciário, pois, onde o processo político falha ou se omite na implementação das políticas públicas e dos objetivos sociais nelas implicados, ou onde direitos sociais são negligenciados por incompetência administrativa, cabe ao Poder Judiciário tomar uma atitude ativa na realização desses fins sociais através da prestação dos serviços sociais básicos (8).

A garantia à saúde e ao direito de receber medicamentos caracteriza-se por ser um direito humano, que se constitucionalizou, tornando-se também um direito social. Não há como o Estado cumprir o dever de garantia do direito à saúde sem alcançar medicamentos aos seus cidadãos (11). A falta de recursos, a escassez de verbas no setor público e a crescente demanda por medicamentos colocam a população em uma situação em que as definições do Executivo ficam aquém das necessidades, e resta como alternativa recorrer ao poder Judiciário para conseguir solucionar as suas necessidades, ou seja, a ideia de judicialização da política diz respeito ao papel que o Judiciário passou a desempenhar nas sociedades contemporâneas como agente ativo na implementação de políticas públicas e efetivação de direitos (8). O Poder Judiciário acaba ganhando espaço para incidir sobre as causas de políticas públicas na área da saúde, gerando o fenômeno da judicialização das políticas de saúde, especialmente no que se refere à assistência médico-farmacêutica em todo o país (12).

Em 2009, devido ao aumento das demandas judiciais, o então Presidente do Supremo Tribunal Federal (STF), Ministro Gilmar Mendes, convocou uma audiência pública onde ouviu 50 especialistas, entre advogados, defensores públicos, promotores e procuradores 
de justiça, magistrados, professores, médicos, técnicos de saúde, gestores e usuários do SUS, com o objetivo de expor as suas contribuições para analisar o fenômeno da judicialização da saúde.

As demandas por medicamentos iniciaram, na década de 90, com os antirretrovirais usados na terapêutica para o HIV/Aids, quando se exigia que o Estado fornecesse o tratamento às pessoas que não tinham condições financeiras para suprir essas necessidades (7), visto que os medicamentos possuíam alto custo e estavam ausentes na lista do SUS. Em 1996, a lei $n^{\circ} 9.313$, em seu art. $2^{\circ}$, obrigou a distribuição gratuita de medicamentos aos portadores do vírus HIV e da Aids, e os recursos retirados do orçamento da Seguridade Social da União, dos Estados, do Distrito Federal e dos Municípios.

A atuação judicial, todavia, deve ocorrer em momento posterior ao da constatação de que as ações positivas estatais não garantiram o direito à saúde. É uma atuação secundária em relação ao dever dos Poderes Públicos (8). A maioria das ações que chegam ao Judiciário pleiteiam intervenções médicas pontuais e específicas, como a realização de determinada operação ou o fornecimento de certo medicamento (13).

Assim, quando a saúde é necessariamente isolada de seu contexto socioeconômico ambiental mais amplo e vista do prisma estreito dos cuidados médicos, grande parte das demandas judiciais se concentra nos processos individuais de cidadãos reivindicando 0 fornecimento de medicamentos. O direito à saúde, no entanto, é um fenômeno amplo e multidimensional, e não apenas o acesso a medicamentos (8):

a intervenção do Poder Judiciário, na administração pública para o fornecimento gratuito de medicamentos procura, entre outras hipóteses, realizar a promessa constitucional da universalidade do SUS. Entretanto, proliferam decisões extravagantes, que condenam a administração ao custeio de medicamentos inacessíveis e ainda, medicamentos experimentais e/ou eficácia duvidosa. Diante disso, os processos terminam por acarretar superposição de esforços e de defesas, envolvendo diferentes entidades federativas e mobilizando grande quantidade de agentes públicos - incluídos procuradores e servidores administrativos. Tudo isso representa gastos, imprevisibilidade e disfuncionalidade da prestação jurisdicional, que põe em risco a própria continuidade das políticas de saúde pública, impedindo a alocação racional dos escassos recursos públicos (8).

Em muitos casos, o que se revela é a concessão de privilégios a alguns jurisdicionados em detrimento da generalidade da cidadania, que continua dependente das políticas universalistas implementadas pelo Poder Executivo (14). 
Nesse contexto o presente trabalho teve por intuito apresentar o panorama municipal das solicitações dos processos judiciais referentes a medicamentos, a partir das observações nos municípios da região metropolitana de Belém-PA, bem como verificar quais os argumentos utilizados pelos autores, juízes e réus em observância da compatibilidade dos processos judiciais com a política pública de medicamentos e quais os tipos de medicamentos solicitados nessas ações.

\section{Metodologia}

A pesquisa teve início a partir do projeto $A$ judicialização da política pública de saúde nos municípios brasileiros, financiado e desenvolvido pelo Hospital do Coração de São Paulo (HCor), em parceria com o Programa de Direito Sanitário da Fiocruz Brasília (Prodisa/Fiocruz Brasília) e o Ministério da Saúde, no âmbito do Programa de Apoio ao Desenvolvimento Institucional do Sistema Único de Saúde (PROADI-SUS).

O projeto foi realizado no estado do Pará, no período de 22 de agosto a 29 de setembro de 2017, em Belém e nos municípios de Marituba e Benevides, na região metropolitana de Belém (PA). A pesquisa caracteriza-se como um estudo descritivo de abordagem quantitativa, por meio da análise de 264 processos de ações judiciais. Desse total, selecionou-se os processos referentes às solicitações de medicamentos. Os dados coletados foram organizados em uma tabela feita no software Excel 2010, tendo como variáveis selecionadas: tipos de autor, principais argumentos utilizados, tipos de solicitações feitas (medicamentos solicitados); tipo de réu; e argumentos utilizados pelo mesmo.

\section{Resultados e discussões}

Durante a pesquisa, foram encontrados 264 processos, com diversas solicitações referentes à saúde. Desses processos, apenas 40 tratavam exclusivamente de medicamentos, visto que vinham com suas prescrições medicas anexadas, critério utilizado para selecioná-los.

Entre essas ações, foram encontrados diversos tipos de autores (Tabela 1) e observou-se um maior número de processos solicitados pela Defensoria Pública (18) e pelo Ministério Público (MP) (19). 
Tabela 1. Representação dos autores das ações judiciais em saúde na região metropolitana de Belém

\begin{tabular}{l|c}
\hline Representação do autor & NM $^{\star}$ \\
\hline Advogado & 3 \\
\hline Defensoria Pública & 18 \\
\hline Núcleo de assistência jurídica & 0 \\
\hline Ministério Público & 19 \\
\hline Total & 40 \\
\hline
\end{tabular}

Fonte: Elaboração própria com base nos dados da pesquisa $A$ judicialização da politica pública de saúde nos municipios brasileiros, do Hospital do Coração de São Paulo (HCor) em parceria com a Fiocruz/Brasília e o Ministério da Saúde.

Nota: NM: número de ações judiciais solicitando medicamentos.

A Constituição Federal (artigo 127) define o Ministério Público como sendo "uma instituição permanente, essencial à função jurisdicional do Estado, incumbindo-lhe a defesa da ordem jurídica, do regime democrático e dos interesses sociais e individuais indisponíveis" (10). A sociedade civil ainda carece de força e organização, sendo incapaz de defender seus direitos fundamentais e, como consequência surge a necessidade de que alguém deva interferir na relação Estado/sociedade em defesa dessa última, ou seja, alguém tem de tutelar os direitos fundamentais do cidadão até que ele mesmo, conscientizado pelo exemplo da ação de seu protetor, desenvolva autonomamente a defesa de seus interesses (15). O MP tem atuado nessa direção, ao fiscalizar a administração pública e atuar na mediação de conflitos e suas resoluções,

Nas questões relacionadas à saúde, o MP tem atuado a partir de ações civis públicas visando o fornecimento de medicamentos, tratamentos, consultas médicas, leitos em UTI, entre outros, em tutela de direito de um único indivíduo ou para uma coletividade de pessoas. Sua atuação é essencial na garantia e efetivação do direito à saúde; e principalmente no que diz respeito a sua legitimidade formal e material para atuar em nome do cidadão, quando este sentir-se lesado devido à omissão ou ineficiência do Poder Público (16).

A Defensoria Pública (DP) é uma instituição permanente, essencial à função jurisdicional do Estado, incumbindo-lhe fundamentalmente, como expressão e instrumento do regime democrático, a orientação jurídica; a promoção dos direitos humanos; e a defesa - em todos os graus, judicial e extrajudicial - dos direitos individuais e coletivos, de forma integral e gratuita (17). 
A DP é a instituição na qual oferece justiça gratuita para aqueles cidadãos que comprovarem não terem condições econômicas de arcar com os custos das ações judiciais concernentes à garantia do direito à saúde. Essa instância tem a sua defesa voltada mais para os interesses individuais (18), enquanto o MP é responsável por zelar pelo ordenamento jurídico, mediando os 70 conflitos existentes entre a sociedade civil e o Estado, suscitado pela não implementação das políticas públicas de responsabilidade do Poder Executivo. Vale enfatizar que a principal defesa do MP está direcionada à sociedade, com vistas a fazer valer os direitos tidos como fundamentais.

Existem diversos motivos pelo qual a sociedade busca o judiciário para a prestação de serviços de saúde, entre eles estão solicitações de consultas medicas, cirurgias, exames, leitos de UTI, medicamentos, produtos de saúde, tratamento de saúde e outros. Nos 264 processos analisados, observou-se que 40 processos $(15,15 \%)$ totalizando 78 medicamentos, desses, seis fármacos foram encontrados mais de uma vez, e com a mesma indicação terapêutica, estes foram analisados e contabilizados uma única vez.

Os argumentos referentes aos medicamentos foram subdivididos, devido à complexidade da assistência farmacêutica no SUS. Nas ações judiciais, foram encontrados medicamentos padronizados, ou seja, que constam nas relações nacionais e municipais de medicamentos essenciais (46 medicamentos); os não padronizados, mas liberados para uso pela Anvisa (25 medicamentos); e o que não possui protocolos clínicos que comprovem sua eficácia e eficiência para o tratamento ao qual foi prescrito (1 medicamento), como mostra a Tabela 2.

Tabela 2. Quantidade de medicamentos solicitados via ação pública de saúde presentes na Região metropolitana de Belém

\begin{tabular}{l|c}
\hline Medicamentos & $\mathbf{N}^{\mathbf{M}^{*}}$ \\
\hline SUS (Rename/Remume) & $\mathbf{4 6}$ \\
\hline Não faz parte da Rename/Remume & 25 \\
\hline Não possui protocolos clínicos & $\mathbf{1}$ \\
\hline Total & $\mathbf{7 2}$
\end{tabular}

Fonte: Elaboração própria com base nos dados da pesquisa $A$ judicialização da politica pública de saúde nos municipios brasileiros, do Hospital do Coração de São Paulo (HCor) em parceria com a Fiocruz/Brasília e o Ministério da Saúde.

${ }^{*}$ Quantidade de medicamentos solicitados. 
A Rename foi criada para ser uma garantia de acesso à assistência farmacêutica e para promoção do uso racional de medicamentos. Sua importância foi reafirmada com a Política Nacional de Medicamentos (PNM), instituída pela portaria $n^{\circ} 3.916$, de 30 de outubro de 1998, e que tem como propósito garantir a segurança, eficácia e qualidade dos produtos utilizados; a promoção do uso racional dos medicamentos; bem como o acesso da população aos medicamentos considerados essenciais. Posteriormente, temos a PNAF, estabelecida pela Resolução n`338 do Conselho Nacional de Saúde, de 19 de setembro de 2003, que engloba, entre outros pontos, o uso da Rename, utilizada periodicamente como instrumento racionalizador das ações no âmbito da assistência farmacêutica, bem como na promoção do uso racional de medicamentos, por intermédio de ações que disciplinem a prescrição, a dispensação e o consumo.

O Decreto no 7.508 , de 28 de junho de 2011 , a partir de seu art. 25, caracteriza a Rename como uma lista de medicamentos selecionados, padronizados e indicados para o atendimento de doenças ou de agravos no âmbito do SUS e que está nas relações específicas complementares (estadual, distrital ou municipal) de medicamentos que somente poderão conter produtos com registro na Agência Nacional de Vigilância Sanitária (Anvisa) (19).

O termo medicamentos essenciais foi conceituado pela Organização Mundial de Saúde (OMS) como sendo aqueles que satisfazem as necessidades de atenção à saúde da maioria da população (20) - observando o seu uso racional -, além de propiciar economia, considerando que os medicamentos selecionados devem ser aqueles com eficácia e segurança comprovadas, ao melhor custo possível.

Dentre os argumentos mais utilizados pelos autores nas ações judiciais para obterem a concessão dos medicamentos, presentes nas Relações ou não, são: o risco de agravar o quadro do paciente; e artigos presentes na Lei $n^{\circ}$ 8.080/90 e na Constituição Federal, em especial o Princípio da Dignidade da Pessoa Humana.

A Constituição Federal, em seus artigos 196 e 197, afirma que a saúde é um direito de todos e dever do Estado, garantindo políticas públicas que visem o acesso universal e igualitário, às ações e serviços para sua promoção, proteção e recuperação, bem como estabelecerem serviços de saúde de relevância pública (10). Já a Lei ㄲo 8.080, de 1990, 
dispõe sobre as condições para a promoção, proteção e recuperação da saúde, a organização e o funcionamento dos serviços correspondentes, e dá outras providências (1). Já na parte contrária da ação (réu), os argumentos mais utilizados nos processos em fase contestatória, foram a perda do objeto da ação; a reserva do possível; a ilegitimidade passiva do Estado; e a grave lesão da ordem social e econômica.

Após a análise dos argumentos apresentados pelas partes da ação em juízo, foi concluído que os pressupostos mais considerados para o deferimento dos pedidos foram: a Constituição Federal, as decisões jurisprudenciais, a Lei n 8.080/1990 e a comprovação e necessidade do pedido. Portanto, a insuficiência da assistência farmacêutica prestada na rede pública, a descontinuidade da prestação do serviço, e mesmo sua omissão, é o que justifica a elevada frequência pelas quais os impetrantes recorrem à Justiça.

Para os medicamentos não incorporados nas listas de medicamentos essenciais do SUS, a Lei n 12.401, de 28 de abril de 2011, estabelece regras específicas de assistência terapêutica e incorporação tecnológica no SUS, sendo necessária a presença de critérios específicos para que um novo medicamento seja incorporado, alterado ou excluído das listas do SUS. Entre os critérios estão as evidências científicas sobre a eficácia; a acurácia; a efetividade e a segurança do medicamento; e a avaliação econômica comparativa dos benefícios e dos custos em relação às tecnologias já incorporadas (21). Essas avaliações são realizadas no SUS pela Comissão Nacional de Incorporação de Tecnologias (Conitec), que reúne, em suas análises, especialistas de diversas áreas do cuidado, representantes das diferentes instâncias de gestão do SUS, além de possuir mecanismos de transparência e participação popular por meio de consultas públicas.

Dos medicamentos não presentes nas relações do SUS, todos têm seu uso permitido pela Anvisa, com a presença de protocolos que confirmam sua eficácia e eficiência, mas a existência de outro medicamento disponibilizado pelo SUS em unidades de saúde municipais e estaduais, pertencente ao mesmo grupo farmacológico e com eficácia, perfil de ação e efeitos colaterais muito semelhantes, permitem que os solicitados nas ações judiciais sejam substituídos pelo outro, sem prejuízo para o tratamento do paciente. A substância fosfoetalonamina, também conhecida como a pílula do câncer, jamais passou por qualquer estudo clínico que comprovasse sua eficácia e segurança e não houve solicitação de registro na Anvisa, regras que todos os medicamentos são obrigados a seguir (22). 
O desenvolvimento de um novo fármaco - molécula ou substância ativa, responsável pelo efeito terapêutico de um medicamento - inicia-se com a descoberta do fármaco e pode ser dividido em três etapas: (a) descoberta de um composto com atividade terapêutica; (a) estudos pré-clínicos; e (c) estudos clínicos (23). Os estudos pré-clínicos têm como objetivo principal a avaliação farmacológica em sistemas in vitro e em animais in vivo para a obtenção do maior conhecimento possível acerca de suas propriedades e de seus efeitos adversos. Além disso, o composto é submetido a testes de toxicidade em animais para que suas propriedades farmacológicas possam ser definidas dentro de uma relação dose-resposta (23).

A fosfoetanolamina não foi submetida a nenhum desses processos, tendo sido utilizada sem esses testes. Para a Anvisa, é um risco sanitário importante haver uma substância com seu uso liberado sem cumprir as exigências regulatórias, visto que a mesma não possui bula, data de validade ou data de fabricação (24).

\section{Considerações finais}

A Constituição Federal de 1988 afirma que a saúde é um direito de todos e dever do Estado, e que deve agir positivamente em prol da população de acordo com suas necessidades. O Sistema Único de Saúde, desde sua criação há 30 anos, vem contribuindo para a melhoria da saúde da população brasileira, mas ainda assim possui dificuldades para a efetivação das suas ações.

O Ministério Público e a Defensoria Pública cumprem com suas atribuições, cujo objetivo principal é atender as necessidades da sociedade relacionadas à saúde, sendo uma instituição onde o usuário será ouvido e onde seu problema será solucionado, em especial as pessoas que não possuem condições financeiras para arcar com os custos das ações judiciais referentes à garantia do direito à saúde.

As ações judiciais, ao solicitarem os medicamentos, fazem uso do arcabouço legal, os três atores - autor, réu e juiz -, em seus argumentos, fazem uso dessas leis para tratar sobre as diversas questões relativas ao sistema de saúde, mas com ênfase nas necessidades dos usuários.

O uso de uma relação de medicamentos tem por objetivo selecionar e padronizar os medicamentos, de modo a garantir uma maior eficácia e segurança para os seus usuários, 
ao melhor custo possível. A solicitação de medicamentos não presentes nas listas dos SUS mostra a falta de conhecimento dos profissionais prescritores, visto que entre os medicamentos prescritos existe uma opção igual nas relações de medicamentos essenciais e que não causará risco ao paciente.

\section{Referências}

1. Brasil. Lei $n^{\circ} 8.080$, de 19 de setembro de 1990. Dispõe sobre as condições para promoção, proteção e recuperação da saúde, a organização e o funcionamento dos serviços correspondentes e dá outras providências. Diário Oficial da União. Brasília. Disponível em: http://www2.camara.leg.br/legin/fed/lei/1990/lei-8080-19-setembro-1990365093-publicacaooriginal-1-pl.html [Acesso em 20.out.2018].

2. Brasil. Ministério da Saúde. Secretaria de Políticas de Saúde. Departamento de Atenção Básica. Política nacional de medicamentos 2001/Ministério da Saúde, Secretaria de Políticas de Saúde, Departamento de Atenção Básica. - Brasília: Ministério da Saúde, 2001. Disponível em: http://bvsms.saude.gov.br/bvs/publicacoes/politica_medicamentos.pdf [Acesso em 20.out.2018]

3. Brasil. Conselho Nacional de Secretários de Saúde. Assistência Farmacêutica no SUS / Conselho Nacional de Secretários de Saúde. Brasília: CONASS, 2007. Disponível em: http://bvsms.saude.gov.br/bvs/publicacoes/colec_progestores_livro7.pdf [Acesso em 20.out.2018]

4. Brasil. Resolução ${ }^{\circ} 338$, de 6 de maio de 2004. Dispõe sobre a Política Nacional de Assistência farmacêutica. Brasília. Disponível em: http://bvsms.saude.gov.br/bvs/saudelegis/cns/2004/res0338_06_05_2004.html [acesso: 20.out.2018]

5. Brasil. PORTARIA № 204, DE 29 DE JANEIRO DE 2007. Regulamenta o financiamento e a transferência dos recursos federais para as ações e os serviços de saúde, na forma de blocos de financiamento, com o respectivo monitoramento e controle. Diário oficial. Brasília. Disponível em: http://bvsms.saude.gov.br/bvs/saudelegis/gm/2007/prt0204_29_01_2007_comp.html [acesso em: 20.out.2018]

6. Maingué Neto W. O Mandado de segurança e o direito à assistência à saúde. Curitiba: Livraria Jurídica e Editora. 2011.

7. Pepe VLD, Ventura M, Sant'ana JMB, Figueiredo TA, Souza VR, Simas L, Castro CGSO. Caracterização de demandas judiciais de fornecimento de medicamentos "essenciais" no Estado do Rio de Janeiro, Brasil. Cad. Saúde Pública, vol 26(3):461-471, Rio de Janeiro, 2010. 
8. Bastos MLC, Sarmento RM. Ações judiciais para o fornecimento de medicamentos no Departamento de Assistência Farmacêutica - PA [Monografia]. Pará: Faculdade de Farmácia, Universidade Federal do Pará; 2012.

9. ALMA- ATA. Cuidados Primários em Saúde. Conferência Internacional sobre Cuidados Primários de Saúde Alma-Ata, URSS, 6-12. Genebra, 1978.

10. Brasil. Constituição da República Federativa do Brasil, 05 de outubro de 1988. Com as alterações determinadas pelas Emendas Constitucionais de Revisão nos 1 a 6/94, pelas Emendas Constitucionais nos 1/92 a 91/2016 e pelo Decreto Legislativo no 186/2008. Brasília: Senado Federal, Coordenação de Edições Técnicas, 2016. Disponível em: https://www2.senado.leg.br/bdsf/bitstream/handle/id/518231/CF88_Livro_EC91_2016.pdf [Acesso em: 20.out.2018]

11. Dauve AC. Responsabilidade do Estado no Fornecimento de Medicamentos e a Intervenção Judicial. Trabalho de Conclusão de Curso, apresentado como requisito parcial para obtenção do grau de Bacharel em Ciências Jurídicas e Sociais da Faculdade de Direito da Pontifícia Universidade Católica do Rio Grande do Sul. Rio Grande do Sul, 2009.

12. Rezende HA. A judicilização dos medicamentos do âmbito do Superior Tribunal De Justiça. Trabalho apresentado ao Centro Universitário de Brasília (UniCEUB/ICPD) como pré-requisito para a obtenção de Certificado de Conclusão de Curso de Pós-graduação Lato Sensu. Brasília 2010.

13. Ferraz OLM, Vieira FS. Direito à saúde, recursos escassos e equidade: os riscos da interpretação judicial dominante. Revista de Ciências Sociais. Vol. 52, no 1pp. 223 a 251. Rio de Janeiro, 2009.

14. Barroso LR. Da falta de efetividade à judicialização excessiva: direito à saúde, fornecimento gratuito de medicamentos e parâmetros para a atuação judicial. Rio de Janeiro, 2007. Disponível em: https://www.conjur.com.br/dl/estudobarroso.pdf [Acesso em: 25.nov.2018]

15. Asensi FD. Indo além da judicialização: o Ministério Público e a saúde no Brasil. Rio de Janeiro: Escola de Direito do Rio de Janeiro da Fundação Getulio Vargas, Centro de Justiça e Sociedade, 2010. 206 p. Disponível em:

http://bibliotecadigital.fgv.br/dspace/bitstream/handle/10438/10313/Indo\%20al\%C3\%A9m\% 20da\%20Judicializa\%C3\%A7\%C3\%A30\%20-

\%200\%20Minist\%C3\%A9rio\%20P\%C3\%BAblico\%20e\%20a\%20sa\%C3\%BAde\%20no\%2 0Brasil.pdf?sequence=1\&isAllowed=y [Acesso em: 25.nov.2018]

16. Barbosa AS, Nakamura FC. A judicialização da saúde e a atuação do Ministério Público no sistema de fornecimento gratuito de medicamentos na região de Ribeirão Preto: estudo sobre a possibilidade de racionalização de recursos materiais e institucionais no 
planejamento de políticas públicas. In: II Seminário Internacional de Pesquisa em Políticas Públicas e Desenvolvimento Social, 2016. UNESP- Franca. Disponível em:

https://www.franca.unesp.br/Home/Pos-graduacao/-

planejamentoeanalisedepoliticaspublicas/iisippedes2016/fernanda---artigo-completosippedes.pdf [Acesso em 25.nov.2018].

17. Brasil. Lei Complementar o 80, de 12 de janeiro de 1994: Organiza a Defensoria Pública da União, do Distrito Federal e dos territórios e prescreve normas gerais para sua organização nos Estados, e dá outras providências. Disponível em:

http://www.planalto.gov.br/ccivil_03/leis/lcp/Lcp80.htm [acesso em 26.nov.2018].

18. Soares AA, Vidal CGM. Direito à saúde, escassez e judicialização: uma tríade de paradoxos [Dissertação]. Paraíba: Departamento de Serviço Social da Universidade Federal da Paraíba, 2017. Disponível em:

https://repositorio.ufpb.br/jspui/bitstream/123456789/3053/1/AAS_CCGMV30102017.pdf [Acesso em: 26.nov.2018].

19. Brasil. Decreto oㅜ 7.508, de 28 de junho de 2011.

Regulamenta a Lei no 8.080, de 19 de setembro de 1990, para dispor sobre a organização do Sistema Único de Saúde - SUS, o planejamento da saúde, a assistência à saúde e a articulação interfederativa, e dá outras providências. Brasília. Disponível em:

http://www.planalto.gov.br/ccivil_03/_Ato2011-2014/2011/Decreto/D7508.htm [Acesso em: 30.nov.2018].

20. World Health Organization. The use of essential drugs: seventh report of the WHO Expert Committee: (including the revised Model List Essential Drugs). Geneva, 1997. (WHO Technical Report Series 867).

21. Brasil. Lei $n^{\circ} 12.401$, de 28 de abril de 2011. Altera a Lei no 8.080 , de 19 de setembro de 1990, para dispor sobre a assistência terapêutica e a incorporação de tecnologia em saúde no âmbito do Sistema Único de Saúde - SUS. Brasília. Disponível em: http://www.planalto.gov.br/ccivil_03/_Ato2011-2014/2011/Lei/L12401.htm [Acesso em: 02.dez.2018]

22. Agência Nacional de Vigilância Sanitária (Anvisa). Fosfoetanolamina. Brasília. Disponível em: http://portal.anvisa.gov.br/resultado-debusca?p_p_id =101\&p_p_lifecycle=0\&p_p_state $=$ maximized\&p_p_mode $=v i e w \& p \_p \_c o l \_i d=$ column-

\&p_p_col_count=1\&_101_struts_action=\%2Fasset_publisher\%2Fview_content\&_101_asse tEntryld=2902065\&_101_type $=$ content\&_101_groupld=219201\&_101_urlTitle=fosfoetanola mina\&inheritRedirect=true [Acesso em: $30 . d e z .2018]$

23. Zebulum JC. O julgamento do caso da fosfoetanolamina e a jurisprudência do Supremo Tribunal Federal. Revista de Direito sanitário. 2017, 17 (3). Disponível em: http://www.revistas.usp.br/rdisan/article/view/127785 [Acesso em: 30.dez.2018] 
24. Costa JD, Alves SMC. Lei da fosfoetanolamina sintética no Brasil. Cadernos IberoAmericanos de Direito Sanitário. 2018 jan./mar, 7(1):215-228. Disponível em: https://www.cadernos.prodisa.fiocruz.br/index.php/cadernos/article/view/468/526 [Acesso em: 30.dez.2018]

Como citar esse artigo:

Damasceno TV, Ribeiro KCB. Judicialização da saúde nos municípios da região metropolitana de Belém-PA. Cadernos lbero-Americanos de Direito Sanitário. 2019 abr./jun.; 8(2): 100-115. 